\section{The Conservation Equations for Multicomponent Gas Mixtures in Arbitrary Coordinate Systems}

F. A. Williams*

Daniel and Florence Guggenheim Jet Propulsion Center. California Institute of Technology, Pasadena, Calif.

February 14, 1958

$\mathbf{T}^{2}$ HE CONSERVATION equations for multicomponent reacting gas mixtures are generally given only in Cartesian or orthogonal curvilinear coordinate systems. Actually, the conservation equations are easily expressed in an arbitrary coordinate system. We present the general equations in tensor notation and then indicate the simplifications which arise for orthogonal curvilinear coordinates.

Let us consider a multicomponent gas mixture consisting of $s$ different species which we identify by Greek subscripts. If $Y_{\alpha}$ is the mass fraction of species $\alpha, \rho$ is the density, $v_{i}$ is the mass average velocity of the mixture, and $V_{\alpha i}$ is the diffusion velocity of species $\alpha$, then the general equations for conservation of mass of component $\alpha$, total mass, momentum, and energy may be written, respectively, as

$$
\begin{gathered}
(\partial / \partial t)\left(\rho Y_{\alpha}\right)+\left(\rho Y_{\alpha} v^{i}\right)_{; i}+\left(\rho Y_{\alpha} V_{\alpha}{ }^{i}\right)_{; i}=\omega_{\alpha} \\
(\partial \rho / \partial t)+\left(\rho v^{i}\right)_{; i}=0 \\
\rho\left(\partial v_{i} / \partial t\right)+\rho v^{j} v_{i ; j}=-P_{i^{j} ; j}+\rho \sum_{\alpha=1}^{s} Y_{\alpha} F_{\alpha i} \\
(\partial / \partial t)(\rho e)+\left(\rho e v^{i}\right)_{; i}=-Q^{i} ; i-P^{i j} v_{i ; j}+\rho \sum_{\alpha=1}^{S} Y_{\alpha} V_{\alpha}{ }^{i} F_{\alpha i}
\end{gathered}
$$

Here $\omega_{\alpha}$ is the mass rate of production of species $\alpha$ per unit volume by chemical reactions, $P_{i j}$ represents the total stress tensor, and $F_{\alpha t}$ is the body force per unit mass acting on the component $\alpha$. In Eq. (4) the quantity $e$ represents the total internal energy of the mixture per unit mass, and $Q_{i}$ denotes the total energy flux vector. English subscripts refer to covariant components, and superscripts identify contravariant components of the tensors.

In Eqs. (1) through (4) use is made of the summation convention for repeated English indices. The semicolon represents covariant differentiation with respect to the coordinates whose

* National Science Foundation Predoctoral Fellow.

† For a discussion of the reasoning by which Eqs. (1) through (4) can be derived, see, for example, P. G. Bergmann, Introduction to the Theory of Relativity, Prentice-Hall, New York, 1942 indices follow it. The covariant derivative of a tensor $A_{i j}{ }^{k}$, for example, is given by

$$
A_{i j}{ }^{k} ; l=\left(\partial A_{i j}{ }^{k} / \partial x^{l}\right)-A_{r j}{ }^{k}\left\{{ }_{i}{ }_{l}\right\}-A_{i r}{ }^{k}\left\{{ }_{j}{ }^{r}\right\}+A_{i j}{ }^{r}\left\{{ }_{r}{ }^{k}\right\}
$$

where the Christoffel symbol of the second kind is defined by the relation

$$
\left\{{ }_{i}{ }_{j}\right\}=(1 / 2) g^{k l}\left[\left(\partial g_{i l} / \partial x^{i}\right)+\left(\partial g_{i l} / \partial x^{i}\right)-\left(\partial g_{i j} / \partial x^{l}\right)\right]
$$

In Eq. (6) the quantity $g_{i j}$ is the metric tensor which defines the coordinate system by means of the expression for the invariant infinitesimal distance.

$$
d s^{2}=g_{i j} d x^{i} d x^{j}
$$

In an orthogonal curvilinear coordinate system the metric tensor is diagonal and we can define the quantities $h_{i}=\partial s / \partial x^{i}$ by the equation

$$
g_{i j}=\left[\begin{array}{ccc}
\left(h_{1}\right)^{2} & 0 & 0 \\
0 & \left(h_{2}\right)^{2} & 0 \\
0 & 0 & \left(h_{3}\right)^{2}
\end{array}\right]
$$

It then becomes convenient to redefine a tensor in such a way that all of its components are of the same physical dimensions. This can be accomplished by dividing each covariant component $i$ of the tensor by $h_{i}$ and multiplying each contravariant component $i$ by $h_{i}$. The new velocities $u_{i}$ and $U_{\alpha i}$, body forces $f_{\alpha i}$, energy flux vector $q_{i}$, and stress tensor $p_{i j}$ are therefore defined by the equations

$$
\left.\begin{array}{l}
v_{i}=h_{i} u_{i} \quad\left[v^{i}=\left(1 / h_{i}\right) u_{i}\right] \\
V_{\alpha i}=h_{i} U_{\alpha i} \\
F_{\alpha i}=h_{i} f_{\alpha i} \\
Q_{i}=h_{i} q_{i} \\
P_{i j}=h_{i} h_{j} p_{i j}
\end{array}\right\}
$$

The summation convention does not apply here nor in the following discussion because of the change in notation. The distinction between covariance and contravariance disappears in this formulation and we shall use subscripts for all indices.

By substituting Eq. (8) into Eq. (6) we find that, in orthogonal curvilinear coordinates, the relation

$$
\begin{array}{r}
\left\{{ }_{i}{ }_{j}\right\}=\left(\delta_{i k} / h_{k}\right)\left(\partial h_{k} / \partial x_{j}\right)+\left(\delta_{j k} / h_{k}\right)\left(\partial h_{k} / \partial x_{i}\right)- \\
\left(h_{i} \delta_{i j} / h_{k}{ }^{2}\right)\left(\partial h_{i} / \partial x_{k}\right)
\end{array}
$$

is valid. Here $\delta_{i j}$ denotes the Kronecker delta. If Eqs. (9) and (10) are used in Eqs. (1) through (4), then the following simplified forms for the conservation equations are obtained:

$$
\begin{gathered}
(\partial / \partial t)\left(\rho Y_{\alpha}\right)+\sum_{i}\left\{\left(1 / h_{i}\right)\left(\partial / \partial x_{i}\right)\left[\rho Y_{\alpha}\left(u_{i}+U_{\alpha i}\right)\right]+\left[\rho Y_{\alpha}\left(u_{i}+U_{\alpha i}\right)\right] \sum_{j \neq i}\left(1 / h_{j} h_{i}\right)\left(\partial h_{j} / \partial x_{i}\right)\right\}=\omega_{\alpha} \\
(\partial \rho / \partial t)+\sum_{i}\left[\left(1 / h_{i}\right)\left(\partial / \partial x_{i}\right)\left(\rho u_{i}\right)+\rho u_{i} \sum_{j \neq i}\left(1 / h_{j} h_{i}\right)\left(\partial h_{j} / \partial x_{i}\right)\right]=0 \\
\rho\left(\partial u_{i} / \partial t\right)+\sum_{j} \rho u_{j}\left\{\left(1 / h_{j}\right)\left(\partial u_{i} / \partial x_{j}\right)+\left(1 / h_{i} h_{j}\right)\left[u_{i}\left(\partial h_{i} / \partial x_{j}\right)-u_{j}\left(\partial h_{j} / \partial x_{i}\right)\right]\right\}= \\
-\sum_{j}\left\{\left(1 / h_{j}\right)\left(\partial p_{i j} / \partial x_{j}\right)+p_{i j}\left(1 / h_{i} h_{j}\right)\left[\left(\partial h_{i} / \partial x_{j}\right)-\left(h_{i} / h_{j}\right)\left(\partial h_{j} / \partial x_{j}\right)+\sum_{k}\left(h_{i} / h_{k}\right)\left(\partial h_{k} / \partial x_{j}\right)\right]-\right. \\
(\partial / \partial t)(\rho e)+\sum_{i}\left[\left(1 / h_{i}\right)\left(\partial / \partial x_{i}\right)\left(\rho e u_{i}+q_{i}\right)+\left(\rho e u_{i}+q_{i}\right) \sum_{j \neq i}\left(1 / h_{j} h_{i}\right)\left(\partial h_{j} / \partial x_{i}\right)\right]= \\
\left.\left.-\sum_{i, j}\left\{\left(p_{i j} / h_{j}\right)\left(\partial u_{i} / \partial x_{j}\right)+\left[\left(p_{j i} / h_{j}\right)-\left(p_{i j} / h_{i}\right)\right]\left(u_{i} / h_{j}\right)\left(\partial h_{i} / \partial x_{j}\right)\right\}+\rho h_{j} / \partial x_{i}\right)\right\}+\rho \sum_{\alpha=1} Y_{\alpha} f_{\alpha i}\left(\sum_{\alpha=1}^{s}\left(Y_{\alpha} \sum_{i} U_{\alpha i} f_{\alpha i}\right)\right.
\end{gathered}
$$

In Eqs. (13) and (14), use has been made of the symmetry of the pressure tensor $p_{i j}$. The derivation of Eqs. (11) through (14) for orthogonal curvilinear coordinates illustrates the method of application of the general conservation equations given in Eqs. (1) through (4). 